\title{
An Improved Multi-Level Screening Identification Algorithm for Lunar Impact Craters
}

\author{
Jiaheng Chen ${ }^{1, a}$, Zhankai Li ${ }^{1, b}$ and Xiaolin Tian"1, 2, c \\ ${ }^{1}$ Faculty of Information Technology, Macau University of Science and Technology, Macao 999078; \\ ${ }^{2}$ Lunar and Planetary Science Laboratory/Space Science Institute, Macau University of Science and \\ Technology, Macao 999078. \\ achenjiaheng1992@126.com, bjanlzk@126.com, cxltian@must.edu.mo
}

Keywords: Lunar impact crater, CCD, recognition algorithm, multi-level.

\begin{abstract}
This study proposes an improved Multi-Level Screening Identification algorithm for the automatic extraction and identification of impact craters based on CCD image. Compared with previous algorithm of Multi-Level Screening Identification, we improved the robustness of algorithm based on terrain classification. First of all, our new algorithm makes a classification of terrain to get the areas of lunar mare and lunar highland and save the classification result by using binary image. At the same time, in order to get the entire suspected craters, our algorithm does the rough extraction and save them as the candidate regions. Next, the algorithm makes the different preprocessing for the different areas respectively according to the binary image of terrain classification. After eliminating many kinds of interference, our algorithm identifies the craters from the different areas as more accurate results. Finally, the algorithm will check the result of identification by the law of light on craters. After these steps, the algorithm can output the counting, the size and coordinates of craters. According to data from the Science and Application Center for Moon and Deepspace Exploration system, we choose the images from H010, Sinus Iridum and Mare Crisium for the testing separately. This algorithm shows higher robustness than previous work and achieves the expected results.
\end{abstract}

\section{Introduction}

The moon is the only natural satellite circulating the Earth. In the 21th Century, the Chang'e Project officially kicked off in China after the successful launches of ShenZhou VI and ShenZhou VII space vessels. Lunar image processing becomes one of the most important aspects of the lunar research. Topography and landform of the moon are the key areas for the moon evolution research. Nowadays, researches on lunar image processing techniques are still in its early stage, most of them are done by manual work. But manual work cannot process the large amount of planet terrain data. Therefore, researchers are trying to use the computer technologies to help them analyze the data.

Several craters make up the main feature of the lunar terrain. Not only the moon, but also Mars and other planets have the same kind of craters on their surfaces. These craters have different sizes, depths and shapes; they are the evidence of planets histories [3]. Without arguing, these craters represent plenty of useful data for us to study the age and history of a planet.

In this thesis, the other researches using conventional methods have also been analyzed and tried. Our new automatic identification procedure is described as below.

\section{Multi-Level Screening Algorithm}

\subsection{The flowchart of improved Multi-Level Screening Algorithm}

From Fig. 1 we can have an overview of our algorithm. Combined with the terrain recognition algorithm, the CCD image will be handled with terrain classification, rough extraction and accurate extraction. Between each level of screening the algorithm will do the appropriate preprocessing to remove interference and improve the recognition rate. The flowchart in Fig. 1 shows the new idea of craters identification. 


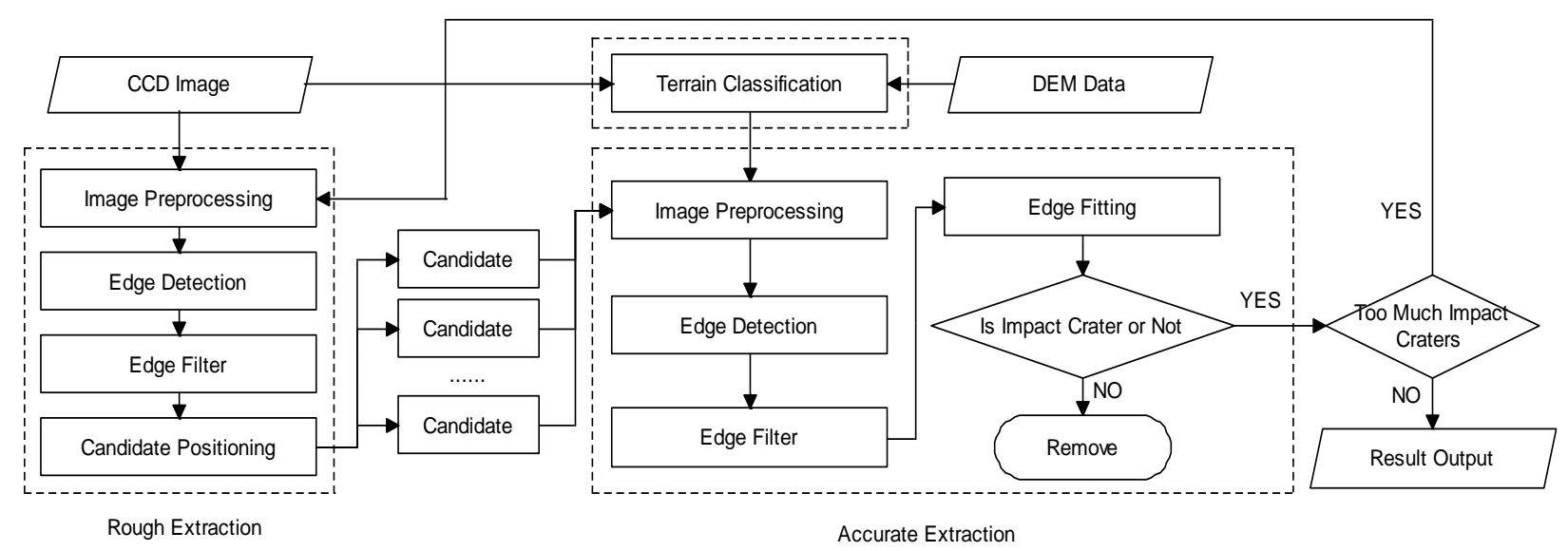

Fig. 1 The flowchart of improved Multi-Level Screening Algorithm

\subsection{The First Screening ---- Terrain Classification.}

As the most obvious feature for a lunar surface, terrain classification is very important to the lunar research [17]. When we analyze a large-sized image, it is necessary to consider the terrain classification. Therefore, we will do terrain classification as our first screening. This screening will help us divide images into different regions and apply different processing steps correspondingly.

During this phase, interference introduced by the difference in terrain classification will be eliminated. As we know, a lunar mare and a lunar highland contain different types of rocks. Different types of rocks always have different light reflectivity and this makes a direct impact on the crater extractions. After a lot of experiments, we determined that the edge extraction and edge fitting algorithms need different thresholds to achieve better results. Therefore, it is necessary to distinguish the lunar mares from lunar highlands.

\subsection{The Second Screening ---- Rough Extraction.}

In this phase, we will extract candidate regions, and apply the image segmentation processing. Rough extraction is designed to reduce the interruption caused by different terrains. After finishing the screening, the candidate regions containing craters will be extracted one at a time. Each candidate region will be processed based on its terrain classification.

The first step in image preprocessing is denoising pre-treatment. In this rough extraction phase, we use histogram stretching and bilateral filtering [4].

Edge detection is a method to automatically detect the lunar craters which is based on mathematical morphology. There are many edge detection algorithms available, and some algorithms have even been entered into the MATLAB libraries directly, for examples, operators like Robert, Sobel, Prewitt, Kirsch, LOG and Canny, etc. For the best result, we were trying to select the best algorithm for the lunar CCD image by repeating the experiment for many, many times.

According to the result of edge detection experiments, we find that algorithms perform differently on different terrains, so we may need to use different ways to process. Some researchers consider that the craters in lunar terrain images are more suitable with Robert Algorithm. Only this algorithm has extracted the necessary information while filtering out a lot of useless information. But it is true that each algorithm needs to have a threshold, some of them use the adaptive threshold, others use fixed threshold [21]. Therefore, maybe we need to manually set a different threshold value of an algorithm for each different CCD image. Otherwise, the adaptive threshold in some algorithm itself is not a good choice; some of them will lead to terrible results. This means sometimes we need to improve the algorithm by ourselves. Actually, many researchers had verified that the Canny algorithm usually has a better performance in edge detection [6].Calculated by Hough algorithm, the candidate regions which contained suspicious of impact craters will be tagged and extracted.

Although Canny operator can ignore most of the noisy edges, it still cannot avoid some false edges [2]. Actually, some edges extracted by Canny are not false edges. Some edges are generated by sun light shadow but we do not want them [1]. In order to solve this problem, we need to filter the false edges by applying the illumination angle. 


\subsection{The Third Screening ---- Accurate Extraction.}

As shown in the flowchart in Fig. 1, the candidate regions will be further extracted with new image sizes during the actual extraction phase. As we know, the image size and the amount of information will impact the result of image processing [8]. Different areas may interact with one another and directly reduce the recognition accuracy.

After the rough extraction phase, each candidate region will have its own characteristic. In this section, we will extract craters from these candidate regions. There are several steps in this phase which we can set the thresholds to get a better result. In addition, different candidate regions have different contrast ratios and different sizes which will all impact both the processing results and the selection of the suitable thresholds. Therefore, we should reduce the searching range to help the algorithm get a better performance through self-adaption [5].

During this phase, some of the processing steps are using the same algorithms used in the previous phase (rough extraction). The steps of edge detection, edge filtering and edge fitting still play an important role in this phase. Finally, the number of extracted craters will influence the processing decision. If the number of extracted craters is acceptable, it will output the result. Otherwise, the program will re-extract the candidate region again.

After the terrain classification, the image is marked by the corresponding tag matrix. In Fig. 2, we can see that each pixel of the terrain image has been marked in a binary image.

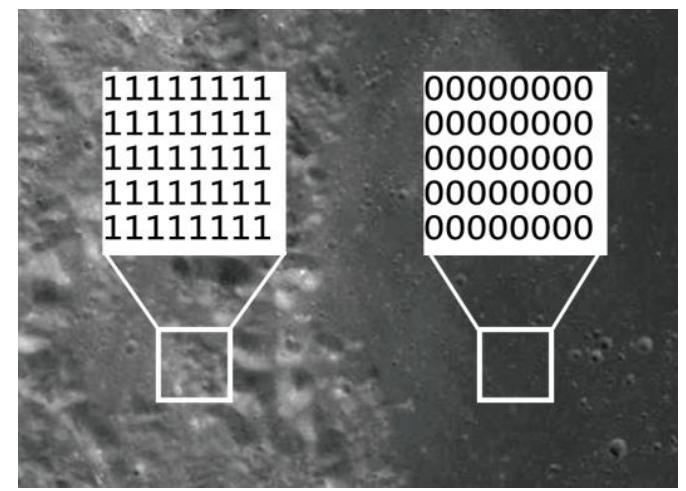

Fig. 2 Image and their corresponding tags matrix

Through these tags in the binary image, we can determine the terrains in the candidate region and change the threshold values accordingly. These thresholds are the optimal solution thresholds which we obtained through the algorithm training using pure lunar mare regions or pure lunar highland regions respectively. For each candidate region, the average value of tags will be used to determine the threshold value. If the average value of tags is closer to 0 , then we let the threshold be set closer to the value of lunar mares, and vice versa.

We can find that the left side of the image is lunar mare, and the right side is lunar highland. The image on lunar mare is obviously smoother than that of lunar highland, and it can be detected with less interference. When we detect the candidate regions on the right side, we need to adjust the threshold closer to the highland threshold value. In this way, both sides can be identified accurately.

\section{Identification Result}

In order to test the identification result on mixed areas, we selected parts of the image to apply the algorithm. In our research, $\mathrm{H} 010$ has the most complex terrain with a mix of lunar mares and lunar highlands. The images we selected have both lunar highlands and lunar mares. Aided by terrain classification results, the results of our algorithm are shown in Fig. 3 and Fig. 4. And the results extracted without terrain classification are compared with our algorithm. 


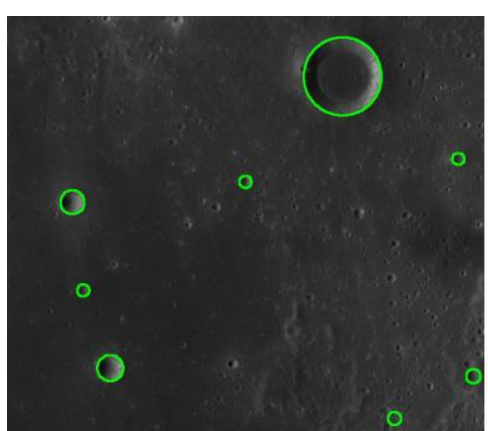

(a)

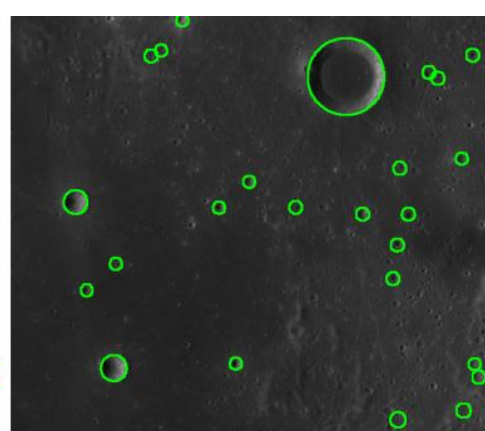

(b)

Fig. 3 The first mixed areas in H010. (a)Extract without terrain classification. (b)Extract with terrain classification.

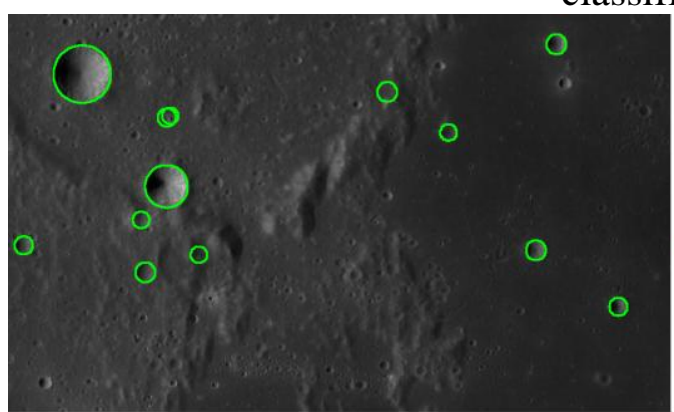

(a)

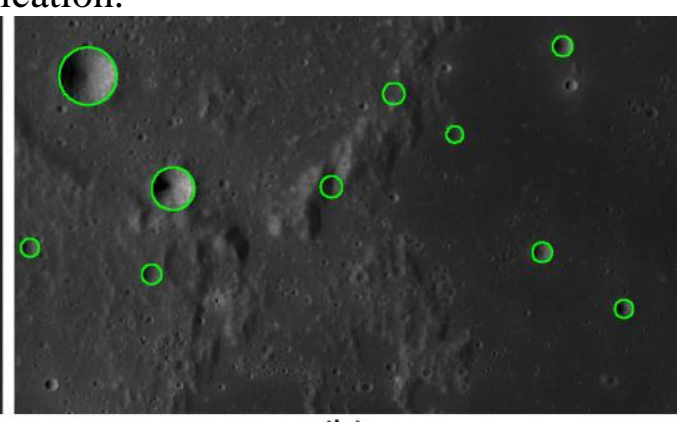

(b)

Fig. 4 The second mixed areas in H010. (a)Extract without terrain classification. (b)Extract with terrain classification.

In these images, we use the classification result to adapt the algorithm to different terrains. As the data in Table 1 shows, the algorithm still produces very good result on the mixed areas. It is obvious that the results with terrain classification have a better accuracy than the previous work done without. However, some of the smaller craters are often being extracted with deviations and it is still hard to identify them accurately.

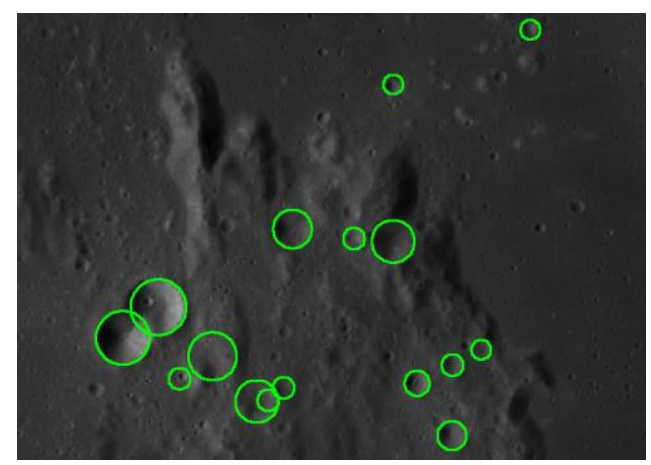

Fig. 5 The third mixed area with complex craters in H010.

In Fig. 5, the mixed areas are still selected from H010. Besides the identification rate, we can also see that the adjacent crates can still be extracted. It shows the robustness of our algorithm which has certain ability to identify complex craters.

Table 1 Result in mixed areas (radius $>=10$ pixels)

\begin{tabular}{ccccc}
\hline Fig. & Manual extraction & Automatic extraction & False extraction & Missed extraction \\
\hline $3($ a) & 19 & 8 & 1 & 8 \\
$3(\mathrm{~b})$ & 19 & 25 & 6 & 0 \\
$4(\mathrm{a})$ & 10 & 13 & 5 & 2 \\
4(b) & 10 & 10 & 2 & 2 \\
5 & 10 & 16 & 6 & 0 \\
\hline
\end{tabular}

\section{Conclusion}

According to the identification results, we can draw our conclusions as follows. 
In most images, the algorithm shows a high accuracy rate on large sized craters. With less interference by other craters, most of the craters can be fitted perfectly. The algorithm not only returns the total number of craters, but also returns the accurate size and locations. In flat areas, small sized craters can still be extracted with high accuracy. In lunar mare areas, our result is always close to the manual extraction result. However, as the image complexity increases, the extraction accuracy rate decreases. The worst results are seen for lunar highland areas. The results of these areas show there are still some weakness in our algorithms. Because of the complexity of terrain characteristic, our extraction results sometimes contain false craters.

After the terrain classification, the accurate extraction can use better threshold values for the preprocessing and the edge detections. In these mixed areas, the accuracy of identification is better than the result in pure highland areas and slightly lower than the result in pure lunar mare areas. Among these results, most of false identifications are caused by the extractions on lunar highlands. However, our work has improved the accuracy of identification in these areas compared with past work. The multi-level screening approach increases the robustness of the identification algorithm.

However, some deficiencies of our algorithm can be concluded as below:

(1) Cannot get a good performance on small size of craters identification.

(2) Cannot get a good performance on blurred areas of high resolution images identification.

(3) The efficiency of processing large size image is very low.

\section{Acknowledgements}

This work is supported by the Science and Technology Development Fund of Macao (No. 059/2013/A2).

\section{References}

[1]. YUAN YueFeng, ZHU PeiMin, ZHAO Na, et al. Automatic identification of circular mare craters based on mathematical morphology. SCIENTIA SINICA Physica, Mechanica \& Astronomica. Vol.43 (2013) No. 3, p. 324-332.

[2]. DU Jun, MIAO Fang, LU Yuhang, et al. Research on appraisal of edge definition of impact craters. Computer Engineering and Applications, Vol. 49 (2013) No. 15, p. 179-183.

[3]. CHEN Ken, LIAO Ping, WANG Yuchuan, et al. Research on Digital Quantitative Classification Method of the Lunar Surface Spots. New Technology \& New Process. Vol. 1 (2015) No. 5, p.106-109.

[4]. Zhizhong Kang, Zhongfei Luo, Teng Hu, et al. Automatic Extraction and Identification of Lunar Impact Craters Based on Optical Data and DEMs Acquired by the Chang'E Satellites. IEEE Journal of Selected Topics in Applied Earth Observations and Remote Sensing. Vol. 8 (2015) No. 10 , p. $4751-4761$.

[5]. JIANG HongKun, TIAN XiaoLin, XU AoAo. An automatic algorithm for detecting lunar impact craters in a defined feature space, SCIENTIA SINICA Physica, Mechanica \& Astronomica. Vol. 43 (2013) No. 11, p. 1430-1437.

[6]. Canny, J., A Computational Approach To Edge Detection [J], IEEE Trans. Pattern Analysis and Machine Intelligence, Vol.8 (1986) No. 6, p. 679-698.

[7]. Zhou Z P, Cheng W M, Zhou C H, et al. Characteristic analysis of the lunar surface and automatically extracting of the lunar morphology based on CE-1 [J]. Chinese Science Bulletin, Vol.56 (2011) No. 9, p. 18-26.

[8]. Zhang Wuming, Zhang Xiaobin, Li Qiaozhi, et al. Automatic Crater Extraction Method for Lunar Images under Low Solar Elevation Angle [J]. Journal of Astronautics, Vol.34 (2013) No. 9, p. 1246-1252. 